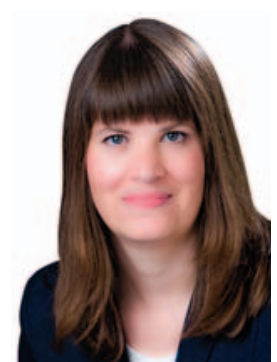

PD Dr. Stephanie Joachim

Koordination «OphthalmoCampus»

\section{Ein Blick über den Tellerrand - Forschung, Weiterbildung und Kommunikation}

Liebe Kolleginnen und Kollegen,

die dritte Ausgabe des «OphthalmoCampus» im Karger Kompass Ophthalmologie beschäftigt sich mit ganz unterschiedlichen Themen, die jedoch alle im Bezug zur Tätigkeit junger Augenärzte und -ärztinnen stehen.

Zum einen stellen wir Ihnen Prof. Dr. Schmitz-Valckenberg von der Universitäts-Augenklinik in Bonn vor, der auf dem EURETINA-Kongress in Kopenhagen im Herbst letzten Jahres mit der «Ophthalmologica Lecture 2016» ausgezeichnet wurde. Sein Forschungsschwerpunkt ist die geographische Atrophie, die nicht-exsudative Spätform der altersabhängigen Makuladegeneration.

Ein Interview mit Teilnehmern des Modellprojektes der Ärztekammer Westfalen-Lippe sowie dem Geschäftsführer dieser Ärztekammer, Dr. Wenning, befasst sich mit der Anerkennung von Forschung in der Weiterbildung. Außerdem wird Ihnen Prof. Molcho die Bedeutung von Körpersprache in der Arzt-Patienten-Beziehung näherbringen. Bei der Kommunikation ist es wichtig, sich nicht in seinen Rollen als Arzt und Patient gegenüberzutreten, sondern von Mensch zu Mensch zu kommunizieren.

Im dritten Teil der Reihe «Wissenschaftliches Publizierens» gibt Dr. Riestenpatt, Projektmanager und Lektor des Karger Verlags, Tipps zur Begutachtung von Artikeln. Diese werden die Erstellung Ihres nächsten, oder vielleicht auch Ihres ersten, Reviews sicher erleichtern.

Für die aktuelle Ausgabe des «OphthalmoCampus» wünsche ich Ihnen viel Spaß beim Lesen!<smiles>CCCCC(C)OCC</smiles> 
Prof. Dr. Steffen Schmitz-Valckenberg

Ophthalmologica Lecture 2016 - The Journey of «Geographic Atrophy» Through Past, Present and Future

Professor Dr. Steffen Schmitz-Valckenberg, stellvertretender Direktor und leitender Oberarzt an der Universitäts-Augenklinik Bonn, wurde auf dem internationalen EURETINA-Kongress in Kopenhagen im September letzten Jahres mit der «Ophthalmologica Lecture 2016» ausgezeichnet. Dieser Preis wird jährlich von der European Society of Retina Specialists (EURETINA) an Wissenschaftler und Forscher verliehen, die das 40. Lebensjahr noch nicht erreicht haben und die wichtige und wertvolle Beträge zur Netzhautforschung geleistet haben. Die Preis-Vorlesung wird im Rahmen des Young Retina Specialists (YouRetina) Symposiums gehalten. Der Preis wird von der S. Karger AG sowohl finanziell als auch durch die Publikation der Preis-Vorlesung in OphtHALmologica, der offiziellen wissenschaftlichen Zeitschrift der EURETINA, gefördert. In seiner Preisvorlesung mit dem Titel «The Journey of ,Geographic Atrophy' Through Past, Present and Future» referierte Prof. Schmitz-Valckenberg über die Erstbeschreibung, das sich mit der Zeit wandelnde Verständnis sowie aktuelle wissenschaftliche und klinische Herausforderungen der nicht-exsudativen Spätform der altersabhängigen Makuladegeneration (AMD).

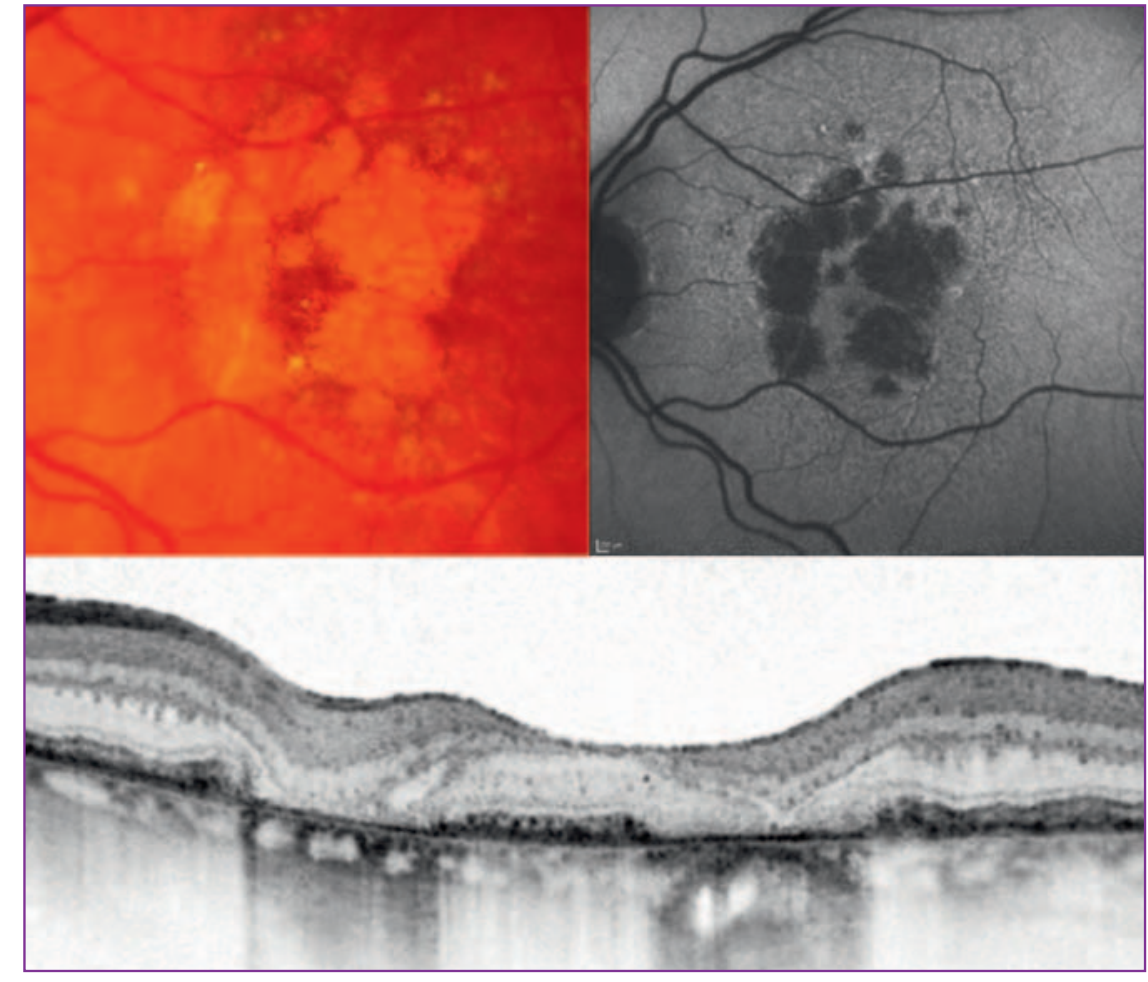

Manifestation einer fortgeschrittenen nicht-exsudativen altersabhängigen Makuladegeneration in multimodaler Bildgebung. Oben links: Fundusfotografie; oben rechts: konfokale Laser-Scan Ophthalmoskopie Fundusautofluoreszenz mit blauem Anregungslicht; unten: Spectral-Domainoptische-Koharänztomografie B-Scan an der Fovea.

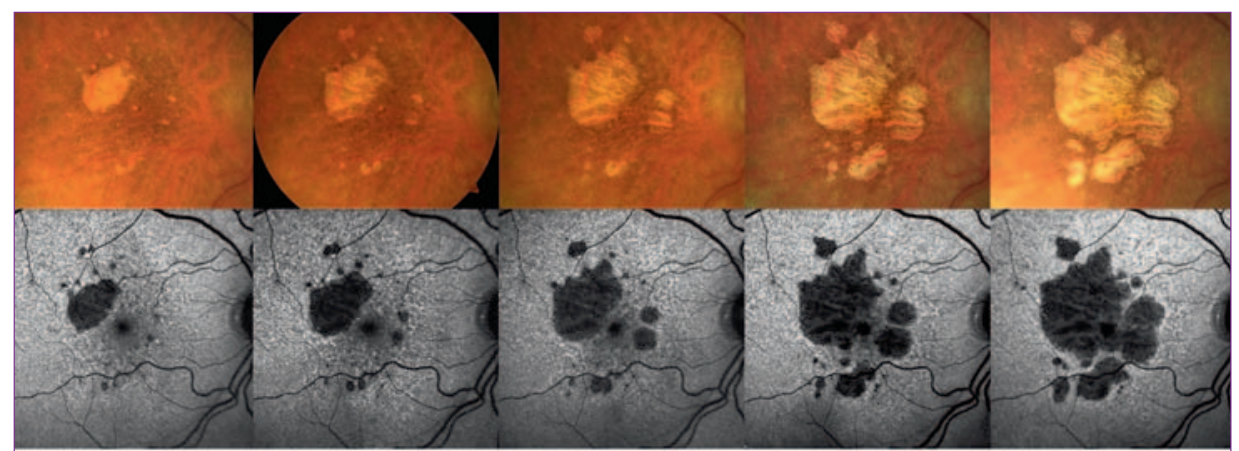

$1 \quad 1.893 \mathrm{~mm}^{2}$

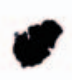

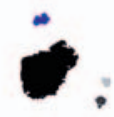

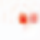

$\begin{array}{llll}2.721 \mathrm{~mm}^{2} & 1 & 3.873 \mathrm{~mm}^{2} \\ 0.180 \mathrm{~mm}^{2} & 2 & 0.432 \mathrm{~mm}^{2} \\ 0.096 \mathrm{~mm}^{2} & 3 & 0.181 \mathrm{~mm}^{2} \\ 0.074 \mathrm{~mm}^{2} & 4 & 0.225 \mathrm{~mm}^{2} \\ 0.073 \mathrm{~mm}^{2} & 5.6 & 0.344 \mathrm{~mm}^{2} \\ 0.067 \mathrm{~mm}^{2} & & \end{array}$

T $1.893 \mathrm{~mm}^{2}$

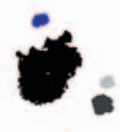

a

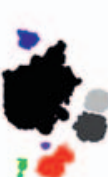

$1 \quad 5.788 \mathrm{~mm}^{2}$

$20.975 \mathrm{~mm}^{2}$

3 $0.622 \mathrm{~mm}^{2}$

$4 \quad 0.387 \mathrm{~mm}^{2}$

$5.60 .926 \mathrm{~mm}^{2}$

$7 \quad 0.143 \mathrm{~mm}^{2}$

$8 \quad 0.060 \mathrm{~mm}^{2}$

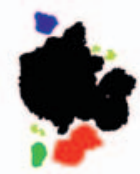

$1 / 21$

$3 / 810.124 \mathrm{~mm}^{2}$

$4 \quad 0.474 \mathrm{~mm}^{2}$

$5.61 .483 \mathrm{~mm}^{2}$

$\begin{array}{ll}7 & 0.373 \mathrm{~mm}^{2}\end{array}$

$9 \quad 0.113 \mathrm{~mm}^{2}$

$10 \quad 0.112 \mathrm{~mm}^{2}$

$110.059 \mathrm{~mm}^{2}$

$12.738 \mathrm{~mm}^{2}$
Entwicklung und Fortschreiten von retinaler Atrophie bei nicht-exsudativer altersabhängiger Makuladegeneration mittels jährlicher Untersuchung über insgesamt 5 Jahre (erste Reihe: Fundusfotografien; zweite Reihe: konfokale Laser-Scanning Ophthalmoskopie Fundusautofluoreszenz mit blauem Anregungslicht). Atrophische Areale wurden halbautomatisch ermittelt und mit der zugehörigen Software quantifiziert (dritte Reihe: zeigt sowohl die Größe der einzelnen Läsionen [in verschiedenen Farben] als auch die Gesamtgröße der Läsion [T]). Zu beachten ist die bessere Ermittlung der Atrophie mit der Fundusautofluoreszenz im Vergleich zur Fundusfotografie, was eine bessere Abgrenzung sowohl der VergröBerung existierender und der Entwicklung neuer einzelner Flecken als auch der Verschmelzung von Flecken über die Zeit hinweg erlaubt. Die Mindestgröße für Läsionen bei der Fundusautofluoreszenz ist auf 0,05 mm² festgelegt. Nach Definition der AREDS (Age Related Disease Study) liegt die Mindestgröße von einzelnen atrophischen Flecken bei 0,145 $\mathrm{mm}^{2}$ (1/8 des Papillendurchmessers), was zu späterer Erfassung atrophischer Satelliten und Bestimmung einer kleineren Gesamtgröße der Atrophie zu verschiedenen Zeitpunkten führen würde. 
Während die «Geographische Atrophie» als nicht-exsudative Spätform der AMD eine häufige Ursache für einen schweren und bisher nicht therapierbaren Sehverlust darstellt und zunehmend - auch aufgrund aufwändiger Forschungsanstrengungen - in den Mittelpunkt rückt, wird der Begriff «Geographische Atrophie» gleichzeitig selbst unter Experten nicht einheitlich verwendet. Damit treten im Alltag Missverständnisse unter Wissenschaftlern und Klinikern, aber auch in der Kommunikation mit Patienten und Angehörigen auf. Die Bezeichnung der Manifestation von Erkrankungen als «geographisch» wurde ursprünglich außerhalb der Augenheilkunde eingeführt. Vor Verwendung im Zusammenhang mit der altersabhängigen (bzw. «senilen», wie ursprünglich bezeichnet) Makuladegeneration wurden Veränderungen der Hornhaut und entzündliche Netzhautveränderungen erstmals durch Donald Gass im Jahre 1970 als «geographisch» beschrieben. Seit Mitte der 1980er Jahre ist



«Der Begriff, Geographische

Atrophie' wird nicht einheitlich verwendet - das führt zu Missverständnissen» der Begriff fest etabliert, allerdings besteht Uneinigkeit u.a. hinsichtlich der genauen Manifestation in Bezug auf betroffene Netzhautschichten und Läsionsgröße, der Ätiologie sowie darüber, ob der Begriff auch im Beisein von exsudativen Veränderungen verwendet werden kann. Insbesondere im Anbetracht zunehmender Fortschritte auf dem Gebiet der hochauflösenden retinalen Bildgebung, der Entschlüsselung von genetischen Veränderungen sowie dem Durchbruch in der Behandlung der neovasuklären AMD mittels VEGF (Vascular Endothelial Growth Factor)-Hemmern werden diese unterschiedlichen Auffassungen und unscharfen Definitionen zunehmend nicht nur für wissenschaftliche Fragestellungen, sondern auch in der alltäglichen Versorgung von Patienten relevant.

Kontaktadresse: Prof. Dr. Steffen SchmitzValckenberg; Universitätsklinikum Bonn AöR, Klinik für Augenheilkunde, Gebäube 05, ErnstAbbe-Straße 2, 53127 Bonn, Deutschland, steffen.schmitz-valckenberg@ukbonn.de

\section{Ärztekammer Westfalen-Lippe \\ Forschung in der Weiterbildung - ein Modellprojekt}

Während der Facharztweiterbildung auch in der Forschung tätig zu sein - und die Zeit auch anerkannt zu bekommen - ist schwer. Seit 2011/2012 setzt sich ein Modellprojekt der Ärztekammer WestfalenLippe für die Anerkennung von Forschungszeiten in der Weiterbildung ein. Nach individueller Vorabprüfung wurden bisher 12 Teilnehmern Forschungszeiten anerkannt, die meisten davon aus der Anästhesie. Aktuell (Stand Juni 2017) sind 8 Weiterbildungsassistenten im Programm. Voraussetzung für die Anerkennung von Forschungszeiten ist das Erstellen eines Zeitplans durch den Weiterbilder, der darstellt, wie trotz Forschungszeiten die Inhalte und Kompetenzen der Weiterbildungsordnung innerhalb der Mindestweiterbildungszeit nach deutscher Weiterbildungsordnung erfüllt werden. Dieses Programm muss vom Vorstand der Ärztekammer genehmigt werden.

Herr Dr. Markus Wenning, geschäftsführender Arzt der Ärztekammer Westfalen-Lippe, Frau PD Dr. Andrea Steinbicker, Oberärztin der Klinik für Anästhesie, operativen Intensivmedizin und Schmerztherapie an der Uniklinik Münster, und Frau Dr. Carolin Kreis, Fachärztin für Unfallchirurgie an der Uniklinik Münster, die beide Teilnehmerinnen dieses Modellprojekts während ihrer Facharztweiterbildung waren, standen uns freundlicherweise Rede und Antwort.

\section{Welche Vorgaben gibt es für das Projekt? Was ist die maximale} Forschungszeit, die anerkannt werden kann?

Dr. Wenning: Rechtliche Rahmenbedingung ist die EU-Richtlinie 2005/36/EG, Anhang V Nummer 5.1.3, die die Mindestzeiten für die Weiterbildung vorgibt. Solange diese eingehalten werden, ist eine Anerkennung der Forschungszeit möglich. Die von der EU vorge- gebene Mindestweiterbildungszeit liegt aber sowieso unter der in Deutschland vorgegebenen. Die Anerkennung von Fremdfächern zeigt auch, dass diese Mindestzeiten Richtwerte sind, die man absenken kann.

Wie gestaltete sich Ihr Forschungsaufenthalt während der Weiterbildung, Frau Dr. Steinbicker?

Dr. Steinbicker: Während meiner Weiterbildung war ich zwei Jahre in den USA. In Amerika habe ich zu 100\% in der Forschung gearbeitet und war im Bereich der Anämieforschung tätig, speziell ging es um molekulare Grundlagen der Eisenregulation und um die Entwicklung künstlicher Blutprodukte. Vorher hatte ich schon ein halbes Jahr in Münster im Labor gearbeitet und parallel Dienste in der Klinik gemacht. Nachdem ich aus den USA zurückgekehrt war, arbeitete ich Vollzeit in der Klinik. Nach dem Abschluss meiner Facharztweiterbildung nahm ich eine Stelle an, die aufgeteilt war in 50\% klinische Tätigkeit und 50\% Forschung. Nach der Berufung zur Oberärztin für Anästhesie am Uniklinikum Münster mache ich weiterhin beides: Ich bin klinisch tätig und leite das Forschungslabor.

\section{Wurde der Forschungsaufenthalt in den USA von Ihrem Chef} unterstützt? Wie wurde er finanziert?

Dr. Steinbicker: Mein Chef an der Uniklinik Münster hat meinen Forschungsaufenthalt sehr stark gefördert. Zusätzlich haben mich die beiden Chefs des Labors in den USA bei der Erstellung eines DFGAntrags auf ein Stipendium unterstützt, durch das ich letztlich den Aufenthalt finanzieren konnte. 
Welche Forschungstätigkeit haben Sie während der Weiterbildung durchgeführt, Frau Dr. Kreis?

Dr. Kreis: Ich komme aus der Unfallchirurgie. In dieser Disziplin habe ich bereits während meines Studiums meine Promotion geschrieben - eine experimentelle Arbeit zur Wirksamkeit von Tigecyclin in der Therapie der Osteomyelitis. Im zweiten Jahr der Weiterbildung habe ich über das Förderprogramm des Interdisziplinären Zentrums für Klinische Forschung Münster (IZKF) eine einjährige Rotationsstelle für das Institut für Klinische Mikrobiologie bekommen. Während dieser Zeit habe ich parallel in Forschung und Klinik gearbeitet, danach wieder Vollzeit in der Klinik. Meine Forschungsprojekte führte ich parallel mit Hilfe von Doktoranden, Drittmitteln und Medizinischen Fachangestellten weiter. Inzwischen bin ich als Fachärztin für Unfallchirurgie zu 100\% klinisch tätig und forsche nebenher.

Dr. Kreis, hatten Sie das Gefühl, dass Ihnen operativ Nachteile entstanden sind, da Sie weniger Zeit im OP verbrachten?

Dr. Kreis: Ich hatte nicht das Gefühl, dass ich praktisch benachteiligt war. Ich habe die Forschungsrotation recht am Anfang meiner Weiterbildung gemacht, zu dem Zeitpunkt, als man an die leichten Eingriffe herangeführt wird. Das konnte ich relativ schnell aufholen. Ich durfte auch in der Forschungszeit selbstständig Dienste machen. Das Gefühl, dass man Nachteile gegenüber Kollegen hat, die nicht in die Forschung gehen, oder dass diese bevorzugt werden, hatte ich nicht.

\section{Wie sind Ihre Erfahrungen mit Anerkennung von Forschungs-} zeit in diesem Modellprojekt?

Dr. Kreis: Bei mir war anfangs noch unsicher, wie viel anerkannt wird, da ich ganz zu Beginn des Modellprojekts in die Forschung gegangen bin. Mir wurde zunächst eine Anerkennung der Hälfte der Zeit zugesichert. Da ich das Forschungsjahr gemacht habe, weil es mir persönlich wichtig war und ich Lust darauf hatte, hätte ich das in Kauf genommen. Als ich dann meine Unterlagen eingereicht habe, hatte sich das Programm aber schon weiterentwickelt und die Forschungszeit wurde vollständig anerkannt.

Dr. Steinbicker: Bei mir war es etwas anders, da ich mit der Forschung insgesamt 7 Jahre in der Weiterbildung war. Ich war das erste Jahr in der Unfallchirurgie tätig und erst im fünften und sechsten Jahr in

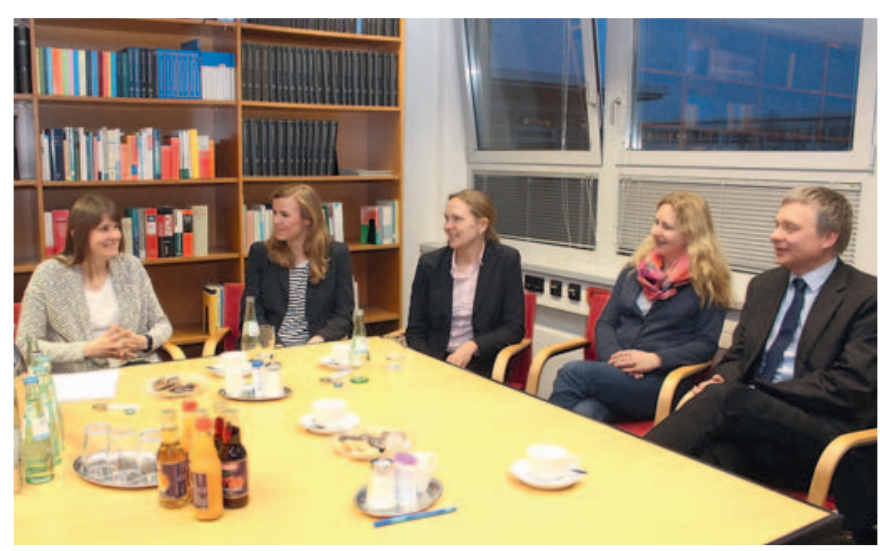

PD Dr. Stephanie Joachim, Dr. Carolin Kreis, PD Dr. Andrea Steinbicker, Bettina Köhler (Ressortleiterin Aus- und Weiterbildung ÄKWL) und Dr. Markus Wenning (v. I. n. r.) im Gespräch. @Pressestelle ÄKWL/Dercks den USA. Das heißt, ich war schon mehr als drei Jahre in der Anästhesie, als ich erfahren habe, dass so etwas anerkannt wird. Es wäre definitiv von Vorteil, wenn man vorab weiß, dass die Forschungszeit anerkannt wird. So verliert man keine Zeit, vor allem, wenn man ein ganzes Jahr in die Forschung will. Ein wichtiger Punkt ist auch die Vergütung der Stellen. Es wäre wichtig, weiter nach TV-Ä bezahlt zu werden, um keinen Nachteil gegenüber den rein klinisch tätigen Kollegen zu haben.

\section{Würden Sie sich wieder für dieses Projekt entscheiden?}

Dr. Kreis: Ja! Für Leute, die sich für die Forschung interessieren, ist es sehr attraktiv und kann nur empfohlen werden. Ich würde raten, es in der ersten Hälfte seiner Weiterbildung zu machen, wenn man in der Klinik eingearbeitet ist und auch schon Dienste machen kann. Dr. Steinbicker: Ich kann es auch nur empfehlen und würde es auch wieder machen. Die Verbindung, die man zwischen Klinik und Labor knüpfen kann, ist sehr wichtig.

\section{Soll dieses Projekt einmal den Modellstatus ablegen? Ist es auch auf andere Ärztekammern übertragbar?}

Dr. Wenning: Wichtiger als die Ärztekammer ist der Weiterbilder. Dieser muss sich zuverlässig kümmern und ein entsprechendes Standing bei seiner Ärztekammer haben, um dort Überzeugungsarbeit leisten zu können. Wichtig ist, dass es eine schriftliche Zusage für die Anerkennung gibt, da man Verlässlichkeit braucht. Diese Zusage gilt dann allerdings nur für die Ärztekammer, von der man das Schreiben bekommen hat. Außerdem ist wichtig, dass der Weiterbilder voll dahinter steht und beispielsweise beim Ausfüllen des Antrags hilft. Auch die Anfrage an die Kammer sollte von ihm ausgehen.

Aus meiner Sicht sind für die Weiterbildung nicht die Zeiten entscheidend, sondern die Kompetenzen. In einem kompetenzbasiertem Modell hat man kein Problem damit, dass Forschung anerkannt wird. Ich hoffe, dass sich das durchsetzen wird.

Dr. Steinbicker: Ich halte es für sinnvoll, erst im zweiten Jahr der Weiterbildung an die Ärztekammer heranzutreten, damit man Zeit hat, sich zu orientieren.

\section{Wie geht es mit dem Modellprojekt weiter?}

Dr. Wenning: Wir sind dabei, dass Clinical Scientist Programme anerkannt werden - an der Uni Münster wird das beispielsweise gerade geplant. Die Anerkennung muss aber individuell erfolgen, um zu prüfen, ob das Vorhaben wirklich umsetzbar ist und ob sich der Weiterbilder wirklich darum kümmert.

\section{Dr. Wenning, Dr. Steinbicker und Dr. Kreis, haben Sie vielen Dank für das Gespräch! \\ Dieses Interview führte PD Dr. Stephanie Joachim für Sie.}

\section{Literatur:}

Wenning M, Van Aken H: Weiterbildung - Forschungstätigkeit und klinische Weiterbildung. Anasthesiol Intensivmed Notfallmed Schmerzther 2016;51:488-489.

Kontaktadresse: Dr. Markus Wenning, Geschäftsführender Arzt der Ärztekammer Westfalen-Lippe, Gartenstraße 210-2014, 47147 Münster, Deutschland, markus.wenning @ aekwl.de 


\section{Von Mensch zu Mensch statt von Arzt zu Patient}

Samy Molcho zählt zu den bedeutendsten Pantomimen des 20. Jahrhunderts und hat die Kunst der Pantomime erstmals um rein psychologische und dramaturgische Elemente erweitert. Seit seinem 10. Lebensjahr ist Molcho mit der Kunst der Darstellung und Interpretation vertraut und nennt die Bühne sein Zuhause. Er studierte klassischen, modernen und fernöstlichen Tanz sowie die Technik der Pantomime in Israel und war seither in beiden Bereichen weltweit sehr aktiv. Das Bewusstsein für die Bedeutung von Körpersprache vermittelt er in entsprechenden Seminaren, die seit Jahrzehnten großen Zuspruch finden.

Sie sind einer der bedeutendsten Pantomimen des 20. Jahrhunderts, bieten aber jetzt Coachings zu Körpersprache für diverse Berufsgruppen an. Bitte skizzieren Sie die zentralen Motive Ihrer Arbeit.

Einer der wichtigsten Aspekte einer zwischenmenschlichen Interaktion, die es immer und überall gibt, sei es mit der Familie, in der Arbeit, oder zwischen Arzt und Patienten, ist der Informationsaustausch. Dabei ist nicht nur wichtig, was der Informationssender von sich gibt, sondern auch wie es auf den Empfänger wirkt und wie er es interpretiert. In der verbalen Kommunikation können wir mehr kontrollieren, was wir sagen und was wir nicht sagen wollen. Nicht so bei der Körpersprache. Oft senden wir durch unseren Körper und unsere Mimik unbewusste Informationen, die der Empfänger richtig oder falsch interpretieren kann - das kann die Kommunikation negativ beeinflussen. Durch das bewusste Einsetzen der Kör-

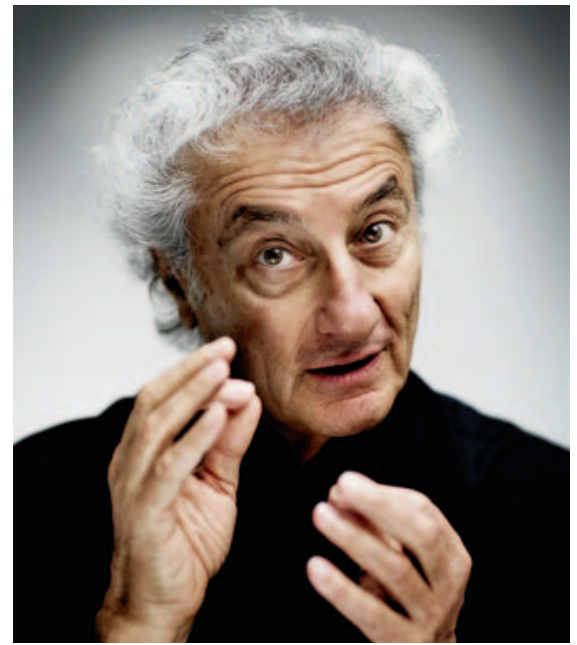

«Respektiere den Patienten zuerst als Mensch und verbessere die zwischenmenschliche Kommunikation.»
Was ist bei der Körpersprache im Arzt-Patienten-Gespräch wichtig?

Auf der medizinischen Ebene gelten nur Symptome und Wissenschaft, dabei kommunizieren aber auch sowohl der Patient als Mensch als auch der Arzt als Mensch. Eine bessere Verständigung auf der zwischenmenschlichen Ebene hilft auch bei den medizinischen Diagnosen und Behandlungen.

Kann man bei der Körpersprache «Fehler» machen? Wenn ja, was wären Beispiele?

Fehler kann man nicht machen, man kann aber unbewusst negative Signale senden. Beispiele:

- eine unzufriedene Miene wegen eines Schreibfehlers in der Akte, was der Patient aber auf sich/seine Diagnose bezieht;

- dominante Befehl-Bewegung mit dem Zeigefinger von oben nach unten;

- Vermeidung von Blickkontakt während eines Diagnosegespräches;

- den Blick auf den Bildschirm zu richten statt auf den Patienten ist eine Missachtung des Patienten, der sich nicht wahrgenommen fühlt.

Weitere Beispiele erfahren Sie in meinen Seminaren.

Worauf sollten vor allem praxisunerfahrene Ärzte achten?

Darauf sollten auch praxiserfahrene Ärzte achten: In der Kommunikation mit Patienpersprache erzielen wir eine höhere Wir-

kung und erwecken Vertrauen in unseren Gesprächspartnern. Es ist wichtig zwischen «wie wirke ich» und «was meine ich» zu unterscheiden. Die Wirkung von Signalen ist oft anders als gemeint.

Welche Rolle spielt Körpersprache in unserer Welt und welche Bedeutung hat Ihre Arbeit für die Gesellschaft im Allgemeinen?

Der Körper sendet ununterbrochen Informationen, ob wir wollen oder nicht. Unabhängig von gesellschaftlichen Spielregeln, von Verhalten und Kommunikation werden innere Empfindungen, die nicht immer Hand in Hand mit diesen Spielregeln gehen, trotzdem gesendet. Sie besser zu verstehen und zu beherrschen, verbessert das zwischenmenschliche Verständnis und dient damit einem besseren und harmonischeren Zusammenleben in unserer Welt. Dazu verhilft uns auch die Kenntnis der Bedeutung von Signalen der Körpersprache in verschiedenen Kulturen und Nationen. So werden Missverständnisse vermieden und mehr Respekt ande- ten von Mensch zu Mensch und nicht von Arzt zu Patient agieren. Kenntnisse der Körpersprache dienen zur Verbesserung dieser Beziehung.

Gibt es einen grundlegenden Rat, den Sie jungen Ärzten mitgeben können?

Respektiere den Patienten zuerst als Mensch und verbessere die zwischenmenschliche Kommunikation, denn dadurch entstehen Sympathien, die helfen, das Vertrauen zwischen Arzt und Patienten zu fördern, was sich im Endeffekt positiv auf die Genesung des Patienten auswirkt.

\section{Prof. Molcho, vielen Dank für das Interview!}

Kontaktadresse: Joram Harel Management, Colloredogasse 32, 1180 Wien, Österreich, office@harel.at, www.samy-molcho.at 


\section{S. Karger Verlag für Medizin und Naturwissenschaften $\mathrm{GmbH}$ Wie begutachte ich einen wissenschaftlichen Artikel?}

Wer Artikel schreibt, zur Publikation einreicht und schließlich veröffentlicht, wird unweigerlich früher oder später von den Journals, in denen er selbst veröffentlicht, gefragt werden, ob er nicht seinerseits ein eingereichtes Paper begutachten kann. Diese Gutachtereinladungen erfolgen in der Regel per Email. Die Gutachten müssen dann innerhalb eines onlinebasierten Einreichungssystems eingegeben werden, das einem meist durch eigene Einreichungen schon bekannt ist. Je nach Journal erfolgt die Begutachtung in einem einfach oder doppelt verblindeten Verfahren. Beim einfach verblindeten Verfahren hat der Gutachter Kenntnis, wer die Autoren der zu begutachtenden Studie sind, beim doppelt verblindeten Verfahren sind auch die Autoren anonymisiert. Sowohl bei dem einen wie auch bei dem anderen Vorgehen wissen aber die Autoren nicht, wer ihre Arbeit begutachtet. Selbstverständlich sind die Gutachter angehalten, Stillschweigen in Hinblick auf die eingereichte Arbeit gegenüber jedem zu wahren, der nicht in den Begutachtungsprozess involviert ist. Im Fall von Interessenskonflikten sollte die Begutachtung in jedem Fall abgelehnt werden. Interessenskonflikte sind z.B.:

- eine Kollaboration mit den Autoren der eingereichten Studie,

- eine direkte Konkurrenzsituation in Hinblick auf die eigene Arbeit,

- eine andauernde Animosität mit den Autoren,

- eigene finanzielle Vorteile, die mit der Publikation des zu begutachtenden Artikels verbunden sind.

Weitere Gründe für eine Ablehnung sind die Unmöglichkeit, das Gutachten innerhalb der vorgegebenen Zeit fertig zu stellen, oder eine völlige Unkenntnis der in der zu begutachtenden Studie behandelten Thematik. In jedem Fall sollten Sie dann dem anfragenden Journal mitteilen, dass Sie das Gutachten nicht übernehmen können.

Bitte bedenken Sie bei einer solchen Ablehnung, dass die grundsätzliche Bereitschaft zur Begutachtung anderer Arbeiten eine tragende Säule des Peer-Review-Verfahrens ist, zu dem es aktuell - zumindest im medizinischen Bereich - keine vernünftige Alternative gibt. Durch die Absage einer Begutachtungsanfrage besteht die Gefahr, dass sich der Begutachtungsprozess einer Arbeit verlängert, und das würden Sie sich ja bei den eigenen Arbeiten auch nicht wünschen.

\section{Wie verfasse ich ein Gutachten?}

In der Regel gibt das jeweilige onlinebasierte Einreichungssystem schon eine Struktur vor. So setzt sich das Begutachtungsformular

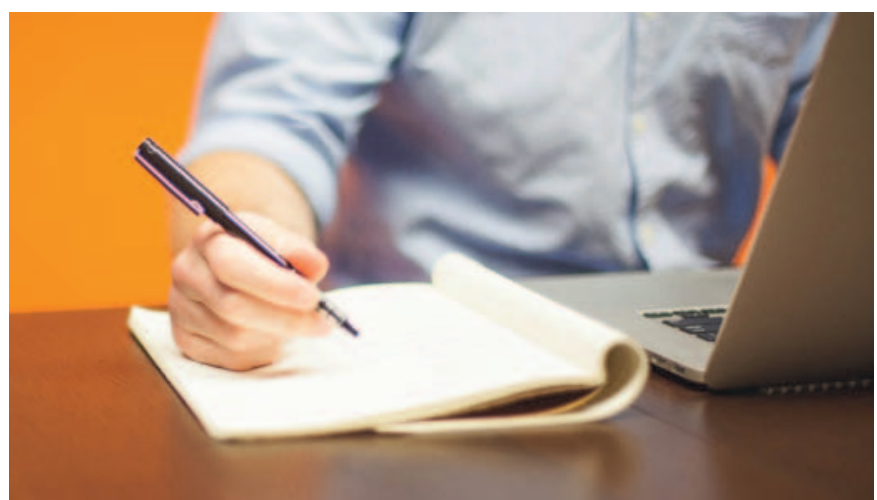

häufig aus einer Reihe von Multiple-Choice-Fragen und einem Kommentarfeld zusammen. Die Multiple-Choice-Fragen ermöglichen es dem zuständigen Associate Editor, schnell einen grundsätzlichen Eindruck von der generellen Einschätzung der Arbeit zu erhalten. Im Kommentarfeld werden Empfehlungen und Korrekturhinweise für die Überarbeitung des Artikels vermerkt, an denen sich der Autor bei der Revision seines Artikels orientieren kann. Grundsätzlich gibt es bei der Begutachtung - ebenso wie beim Schreiben eines Artikels - eine Reihe von Grundsätzen, die beachtet werden sollten. Ein hilfreiches Gutachten sollten folgende Fragen beantworten:

- Präsentiert die Arbeit neue Befunde?

- Ist die Arbeit von Bedeutung für Forscher und Kliniker in diesem Fachgebiet?

- Ist die Arbeit von Bedeutung für Forscher und Kliniker außerhalb des Fachgebiets?

- Sind Methoden und statistische Verfahren in angemessener Weise verwendet worden?

- Wurden sämtliche ethische Standards berücksichtigt?

- Sind die Schlussfolgerungen der Autoren evidenzbasiert?

- Enthält die Arbeit falsche Informationen oder sind wichtige Dinge nicht berücksichtigt?

- Ist die aktuelle Literatur angemessen zitiert?

- Ist die Arbeit gut geschrieben, klar und leicht zu verstehen?

- Wenn die Arbeit Tabellen oder Abbildungen enthält, helfen diese dem Leser oder sind sie überflüssig?

\section{Was geschieht nach der Abgabe des Gutachtens?}

Bei einem ordentlichen Peer-Review-Verfahren gibt es zu jeder Arbeit mindestens zwei Gutachten. Liegen die Gutachten vor, wird der zuständige Associate Editor auf der Basis der Gutachten um eine abschließende Entscheidung gebeten. Weichen die Gutachten stark voneinander ab, kann er entweder selbst ein weiteres Gutachten anfertigen oder um die Anfrage eines dritten Gutachters bitten. Im Anschluss wird der Autor über die Entscheidung informiert und erhält in diesem Zuge auch die anonymisierten Gutachten. Im Falle einer Überarbeitung wird der Autor gebeten, innerhalb eines definierten Zeitraums die revidierte Fassung seines Artikels zusammen mit einem detaillierten Antwortbrief zu den Gutachterkommentaren erneut einzureichen. Der zuständige Associate Editor prüft dann die Revision; gegebenenfalls kann es auch nochmals die Gutachter um ihre Meinung bitten. Viele Journals informieren die Gutachter nach Abschluss des Begutachtungsprozesses über die finale Entscheidung (Annahme/Ablehnung). Häufig werden den Gutachtern dabei die jeweils anderen Gutachten zu ihrer Information zur Verfügung gestellt.

Kontaktadresse: Dr. Sven Riestenpatt, Projektmanagement \& Editorial Office Transfusion Medicine and Hemotherapy / Obesity Facts, Karger Verlag GmbH S. Karger Verlag für Medizin und Naturwissenschaften GmbH, Wilhelmstraße 20a, 79098 Freiburg, Deutschland, s.riestenpatt@karger.com 